\title{
The comparison of cold water immersion and cold air therapy on maximal cycling performance and recovery markers following strength exercises
}

Kane J Hayter, Kenji Doma, Moritz Moritz Schumann, Glen B Deakin

This study examined the effects of cold water immersion (CWI) and cold air therapy (CAT) on maximal cycling performance (i.e. anaerobic power) and markers of muscle damage following a strength training session. Twenty endurance-trained but strength-untrained male $(n=10)$ and female $(n=10)$ participants were randomised into either: CWI (15 minutes in $14^{\circ} \mathrm{C}$ water to iliac crest) or CAT ( 15 minutes in $14^{\circ} \mathrm{C}$ air) immediately following strength training (i.e. 3 sets of leg press, leg extensions and leg curls at 6 repetition maximum, respectively). Creatine kinase, muscle soreness and fatigue, isometric knee extensor and flexor torque and cycling anaerobic power were measured prior to, immediately after and at 24 (T24), 48 (T48) and 72 (T72) hours post-strength exercises. No significant differences were found between treatments for any of the measured variables $(p>0.05)$. However, trends suggested recovery was greater in CWI than CAT for cycling anaerobic power at T24 (10\% $\pm 2 \%, \mathrm{ES}=0.90), \mathrm{T} 48(8 \% \pm 2 \%, \mathrm{ES}=0.64)$ and T72 $(8 \% \pm$ $7 \%, E S=0.76)$. The findings suggest the combination of hydrostatic pressure and cold temperature may be favourable for recovery from strength training rather than cold temperature alone. 
1 The comparison of cold water immersion and cold air therapy on maximal cycling

2 performance and recovery markers following strength exercises

3

4 Short title: Effects of cold water immersion on recovery following strength training

5

\section{Authors}

7 Kane J. Hayter ${ }^{1}$, Kenji Doma ${ }^{2}$, Moritz Schumann ${ }^{3}$ and Glen B. Deakin ${ }^{1}$

8

\section{Affiliations}

${ }^{1}$ Sport and Exercise Science, James Cook University, Cairns, Australia

$11{ }^{2}$ Sport and Exercise Science, James Cook University, Townsville, Australia

$12{ }^{3}$ Faculty of Sport and Health Sciences, Department of Biology of Physical Activity, University

13 of Jyväskylä, Finland

Corresponding Author:

Kenji Doma PhD

171 James Cook Drive, Rehab Sciences Building (DB-43), James Cook University

QLD 4811

19 Australia

20 Phone (07) 47814952

21 Email: kenji.doma@jcu.edu.au 


\section{Abstract}

23 This study examined the effects of cold water immersion (CWI) and cold air therapy (CAT) as

24 recovery modalities following a strength training session on maximal cycling performance (i.e.

25 anaerobic power) and markers of muscle damage. Twenty endurance-trained but strength-

26 untrained male $(\mathrm{n}=10)$ and female $(\mathrm{n}=10)$ participants were randomised into either: CWI $(15$

27 minutes in $14^{\circ} \mathrm{C}$ water to iliac crest $)$ or CAT $\left(15\right.$ minutes in $14^{\circ} \mathrm{C}$ air $)$ immediately following

28 strength training (i.e. 3 sets of leg press, leg extensions and leg curls at 6 repetition maximum,

29 respectively). Creatine kinase, muscle soreness and fatigue, isometric knee extensor and flexor

30 torque and cycling anaerobic power were measured prior to, immediately after and at 24 (T24),

3148 (T48) and 72 (T72) hours post-strength session. No significant differences were found

32 between treatments for any of the measured variables $(p>0.05)$. However, trends suggested

33 recovery was greater in CWI than CAT for cycling anaerobic power at $\mathrm{T} 24(10 \% \pm 2 \%, \mathrm{ES}=$

$340.90), \mathrm{T} 48(8 \% \pm 2 \%, \mathrm{ES}=0.64)$ and $\mathrm{T} 72(8 \% \pm 7 \%, \mathrm{ES}=0.76)$. The findings suggest that the

35 combination of hydrostatic pressure and cold temperature may be favourable for recovery from

36 strength training rather than cold temperature alone. 
38

Introduction

A growing body of evidence suggests that the application of cold water immersion (CWI) following strength exercise may accelerate recovery to alleviate symptoms of delayed onset of muscle soreness (DOMS) and muscle damage (Leeder, Gissane, van Someren, Gregson, \& Howatson, 2012). The reported benefits include peripheral vasoconstriction (Karunakara, Lephart, \& Pincivero, 1999) which increases metabolite removal (Cochrane, 2004) and a decrease in oedema formation (Dolan, Thornton, Fish, \& Mendel, 1997; Kowal, 1983).

Of the studies that have examined CWI effects following strength exercises, the comparator groups have typically involved active recovery (Roberts, Nosaka, Coombes, \& Peake, 2014), warm water immersion (Vaile, Halson, Gill, \& Dawson, 2008) and contrast therapy (i.e. alternating between warm and cold water) (Vaile et al., 2008). Whilst these conditions demonstrate the influence of temperature on recovery, it does not account for contribution of hydrostatic pressure during water immersion.

One method of accounting for hydrostatic pressure on recovery could be to compare recovery dynamics between hydrotherapy (i.e. in cold water) to non-hydrotherapy (i.e. in cold air) conditions. Recent studies have reported that cryostimulation (i.e. exposure to cold air) at extremely cold conditions (e.g. under $-100^{\circ} \mathrm{C}$ ) may accelerate recovery and minimise inflammatory responses responsible of inducing DOMS following muscle damaging exercises (Ziemann et al., 2014) and mitigate signs of overtraining during periods of intense training (Schaal et al., 2015). However, the typical length of exposure for cryostimulation is approximately 2-3 minutes (Costello et al., 2015) due to the extreme conditions, which is substantially less than the typical exposure for CWI of 10-15 minutes (Versey, Halson, \& 
60 Dawson, 2013). Subsequently, comparisons for the effect of recovery between the conventional 61 method of cold air treatment (CAT) and CWI is at present difficult due to large differences in

62 technique. However, by standardizing the temperature between CWI and CAT (e.g. $4^{\circ} \mathrm{C}$ in both

63 hydrotherapy and non-hydrotherapy conditions), the length of exposure between treatments

64 could be equated thereby providing the opportunity to determine the contribution of hydrostatic

65 pressure during CWI. To date, comparison between such conditions is limited, particularly

66 following a typical strength training session.

67 Furthermore, it is unknown whether the benefits of CWI are also reflected in sprint-based

68 exercises (i.e. anaerobic performance) days following a typical strength training session,

69 particularly in strength- untrained and/or detrained individuals where DOMS may elevate to

70 levels that may impair anaerobic performance. Several studies have reported impaired running

71 (Doma \& Deakin, 2013b, 2014; Doma et al., 2015; Twist \& Eston, 2005) and cycling (Byrne \&

72 Eston, 2002; Nieman et al., 2014) performance at maximal intensities for 24-72 hours post

73 strength exercises in strength-untrained individuals. These findings have severe implications for

74 the quality of maximal intensity intermittent training sessions when combined with strength

75 training sessions in the one program, also known as concurrent training (Hickson, 1980). In fact,

76 Doma and colleagues (2015) recently showed that the combination of alternating-day strength

77 training and consecutive-day maximal intensity endurance training impaired running

78 performance at maximal effort over the course of a typical micro-cycle of concurrent training,

79 which may possibly be detrimental to optimal long-term adaptations. Subsequently,

80 incorporating recovery modalities, such as CWI, may alleviate acute carry-over effects of fatigue

81 in-between each mode of training session and thereby optimise the quality of high intensity

82 intermittent training sessions. Rowsell and colleagues (2014) previously reported recovery of 
83 maximal cycling time-trial and cycling interval 9 hours following run training via use of CWI,

84 suggesting that CWI may in fact accelerate recovery and minimise carry-over effects of fatigue

85 on maximal intensity intermittent training sessions several hours post strength training. However,

86 little is known whether benefits of CWI for maximal sprint-based performance are present

87 following typical strength exercises (e.g. leg press, leg extension and leg curls), particularly

88 during periods when DOMS peak (i.e. several days post).

89 The purpose of the current study was to compare the effect of CWI and cold air treatment (CAT)

90 on maximal cycling performance and post-exercise markers of muscle damage following a

91 typical strength training session in endurance-trained but strength-untrained individuals. 
96

97 Research design

98 The study was conducted over a 2 week period (Figure 1) with subjects attending a ***Figure 1 around here***

Methods familiarisation session followed by 4 testing sessions. The familiarisation session occurred in the first week and allowed participants to become familiar with the testing procedures and equipment as well as completing a 6 repetition maximum (6RM) assessment. The 6RM assessments followed previously described guidelines (Baechle \& Earle, 2008) for incline leg press (Maxim, MPL 701, Adelaide, Australia), leg extensions and leg curls (Maxim, P 5021, Adelaide, Australia). After a minimum of 4 days of rest, the participants returned to the laboratory and completed a strength training session. Indirect markers of muscle damage, muscle force generation capacity and maximal cycling performance were measured prior to (T0) and immediately post (T1) as well as 24 (T24), 48 (T48) and 72 (T72) hours post the strength training session. Immediately following the T1 testing time point, participants undertook the recovery protocol either as an intervention by submerging into water (i.e., CWI) or as a CAT. During each visit, participants underwent a standardised warm-up consisting of five minutes of stationary cycling (Monark, Ergomedic 828E, Sweden). Biological variations were controlled by conducting the strength training sessions at the same time of day, refraining from caffeine or food intake at least 2 hours prior to testing and high intensity physical activity for a minimum of 24 hours prior.

Subjects 
117 Twenty strength-untrained but moderately endurance trained males $(n=10)$ and females $(n=10)$

118 volunteered to participate in this study. The participants were recreational endurance trained

119 males and females (e.g. runners, cyclists) who had been participating in moderate-high intensity

120 endurance exercise at least twice a week for the previous 12 months and had not performed

121 lower body strength trainings sessions for at least 6 months. The participants were manually

122 matched paired by gender, age and muscular strength and then allocated at random to either a

123 cold water immersion $(\mathrm{CWI}$; age $25.3 \pm 6.0$ years, height $170.9 \pm 8.1 \mathrm{~cm}$, body mass $70.2 \pm 8.9$

$124 \mathrm{~kg}$, leg press strength $169.5 \pm 71.7 \mathrm{~kg}$ ) or CAT (age $22.5 \pm 3.9$ years, height $171.6 \pm 10.5 \mathrm{~cm}$,

125 body mass $70.4 \pm 13.9 \mathrm{~kg}$, leg press strength $167 \pm 63.3 \mathrm{~kg}$ ) group and were matched by gender,

126 age and muscular strength. Whilst previous CWI studies have used a cross-over design (Jajtner et

127 al., 2015; Roberts et al., 2014), given that the purpose of the current study was to examine the

128 recovery effects of CWI and CAT in strength-untrained individuals, we separated participants

129 into groups to avoid repeated bout effect or learning effects (Doma et al., 2015). Before

130 commencing the study, each participant provided their written informed consent and did not

131 report illness, disease and injury or medication that would contraindicate any protocols that were

132 approved by the James Cook University Human Research Ethics Committee (HREC; Approval

133 number H5565).

135 Procedures

136 Strength Training Session

137 The selection of exercises, intensity and duration of the strength training session was adapted

138 from previous studies (Doma \& Deakin, 2013a, 2014) including leg-press, leg extensions and leg 
139 curls. These exercises were selected to replicate a typical lower body strength training session

140 targeting maximal strength development (i.e. a low number of repetitions with high loads),

141 typically recommended for endurance-trained subjects. In order to standardize the technique of

142 each exercise, machine-based equipment was used. Specifically, participants underwent a warm-

143 up set prior to their first working set of the strength training session by performing 10 repetitions

144 of incline leg press using half the load of their 6RM. Participants then completed 5 sets of 6

145 repetitions of leg press at 6RM load and 3 sets of 6 repetitions of leg extensions and leg curls,

146 both at 6RM load. A three minute recovery was provided between each set and each exercise.

\section{Maximal cycling performance}

149 A 10-second Wingate test was performed on a bicycle ergometer (Velotron, Racermate, USA)

150 using a standard protocol (Minett et al., 2013). Whilst a performance test of a longer duration, or

151 over multiple sets, would have been desired, this cycling duration of a single set was selected to

152 avoid additional fatigue across the post-strength training days (i.e. T24, T48 and T72).

153 Furthermore, this duration has been used over a number of sets during high intensity intermittent

154 cycling training to induce anaerobic capacity and improve sprint-ability (Tabata et al., 1996;

155 Wells, Edwards, Fysh, \& Drust, 2014). Subsequently, attenuation of such performance measure,

156 even with a single set, due to preceding strength exercise-induced fatigue would suggest

157 impairment of the quality of high intensity intermittent training sessions. Specifically,

158 participants cycled at 50W for 20 seconds, then cycled with the absence of any resistance for 5

159 seconds before finally cycling against a weighted brake (i.e., torque values of 0.087 and 0.084 
160 for males and females, respectively) for 10 seconds. The participants were verbally encouraged

161 during the protocol to ensure that cycling was executed at maximal effort.

162

163

164

165

166

167

168

169

170

171

172

173

174

175

176

177

178

179

180

\section{Indirect Markers of Muscle Damage}

Indirect markers of muscle damage were recorded via blood sampling (i.e., creatine kinase [CK]) and perceptions of muscle soreness and fatigue. Prior to analysing CK, calibration of the analyser (Reflotron Plus, Roche Diagnostics, Australia) was completed in accordance with the instructions of the manufacturer. Capillary blood samples were then collected using a finger prick method to analyse CK. Ratings of muscle soreness and fatigue were obtained following the completion of three repetitions of body weight squats. The muscle soreness and fatigue rating method used in this study was a standard 1-100 visual analogue scale adapted from a previous study (Doma \& Deakin, 2015). Specifically, 1 to 100 indicated "not very sore at all" to "very sore", respectively, for the rating of muscle soreness and 1 to 100 indicated "not fatigued at all" to "very fatigued", respectively, for the rating of muscle fatigue.

\section{Muscle Force Generation Capacity}

Maximum voluntary isometric contraction (MVC) testing was performed using a custom built isometric dynamometer chair (James Cook University, Cairns, Australia). The isometric dynamometer chair was set so as to position the right knee at an angle of $110^{\circ}$. A force

transducer was placed superior to the right medial and lateral malleoli and calibrated by placing a known weight against the transducer. A high reliability $(\mathrm{ICC}=0.76-0.95$, mean 0.85$)$ and minimal variability $(\mathrm{CV}=4.1-5.9 \%$, mean $5.1 \%)$ has been reported previously with this chair (Doma \& Deakin, 2014). Participants were given three attempts at leg extensions and leg curls 
181 and were required to keep the maximal force plateaued for 6 seconds. A 90 -second rest was 182 allowed between attempts. The trial with the highest torque was then reported.

183 Recovery Protocol

184 Participants in the CWI group sat in an inflatable bath (White Gold Fitness, UK) containing 185 water at $14^{\circ} \mathrm{C}$ for 15 minutes. To ensure that the lower extremity was fully submerged, the knees 186 were extended and the water level was set to the iliac crest. To maintain the constant temperature 187 of the water it was monitored using a thermometer and stirred at 5-minute intervals with more ice 188 added if necessary. The CAT group sat in the same bath without water in a custom-built climate 189 chamber (James Cook University, Townsville, Australia) set at $14^{\circ} \mathrm{C}$ and $60 \%$ humidity for 15 190 minutes. This humidity matched the ambient humidity that the CWI group were exposed to 191 during the recovery intervention. Once positioned in the bath, blood pressure, heart rate and 192 rating of thermal comfort were recorded at three minute intervals (i.e. five times) for safety 193 monitoring purposes. Thermal comfort was recorded using a 1 to 5 scale indicating 194 “comfortable" to "extremely uncomfortable", respectively (Minett et al., 2013).

The measure of central tendency and dispersion are reported as mean \pm standard deviation.

198 Given that the study incorporated male and female participants, inter-individual variability was controlled for by quantifying percentage differences from T1, T24, T48 and T72 against T0 for peak watts, mean watts and total work from the 10 -second Wingate cycling test. The ShapiroWilk test revealed that all data analysed were normally distributed. Therefore, a two-way (time $\mathrm{x}$ group) repeated measures analysis of variance (ANOVA) was used to determine differences 
203 between each time point and between the CWI and CAT groups for the dependent variables with

204 gender as a covariance. Bonferroni adjustments were performed for pairwise comparisons where

205 significance was found. Significance was reached at an alpha level of $p \leq 0.05$. Effect size (ES)

206 was calculated to determine the magnitude of differences between the CWI and CAT groups for

207 each time point (i.e. T0, T1, T24 and T48) using the formula:

$208 \mathrm{ES}=(\mathrm{CWI}-\mathrm{CAT}$ mean $) /$ standard deviation $($ Rhea, 2004)

209 Between-group ES for the indirect markers of muscle damage (i.e. CK, muscle soreness, muscle

210 fatigue, KET and KFT) was calculated based on absolute measures. For the maximal cycling

211 performance measures (i.e. peak watts, mean watts and total work), between-group ES was

212 calculated based on the normalised data (i.e. percentage change across time). ES calculations

213 were interpreted as trivial effect at $<0.2$, small effect at $0.2-0.49$, moderate effect at $0.5-0.79$ and

214 a large effect at $>0.8$ (Cohen, 1988). All data were analysed using the Statistical Package for

215 Social Sciences (SPSS, version 22, Chicago, Illinois). 
217 Results

218 There were no significant differences between groups for age and strength levels $(\mathrm{p}>0.05)$

219 indicating that the two groups were successfully matched for these parameters. Gender as a

220 covariate showed no significant effects for all measures $(p>0.05)$ except for a time $\mathrm{x}$ gender

221 interaction effect $(\mathrm{p}<0.05)$ for $\mathrm{CK}$, suggesting that gender did not influence the majority of the

222 outcome measures for the two groups.

223 Maximal cycling performance

224 A main effect for time was found for mean watts $(\mathrm{p}<0.01)$ and total work $(\mathrm{p}<0.01)$ but not

225 peak watts $(\mathrm{p}>0.05$; Table 1$)$. Similarly, these parameters showed no effect of time when

226 examined per group (Table 2$)$ with no time $x$ group interaction effect $(p>0.05)$. However, effect

227 size calculations between groups showed moderate to large differences between the groups

228 (Figure 2). At T24, a large difference was found between groups for peak watts, mean watts and

229 total work. Furthermore, a moderate difference was found between groups at T48 and T72 for

230 peak watts while a large difference was found at T48 and moderate difference at T72 for mean

231 watts.

$232 * * *$ Table 1 around here***

$233 * * *$ Table 2 around here***

$234 * * *$ Figure 2 around here***

235

236

Indirect Markers of Muscle Damage

237 A main effect for time was found for $\mathrm{CK}$, muscle soreness, muscle fatigue and knee extensor

238 torque $(\mathrm{p}<0.01)$ but not for knee flexor torque $(\mathrm{p}>0.05$; Table 3$)$. Similarly, a main effect of 
239 time was found for $\mathrm{CK}$, muscle soreness, muscle fatigue and knee extensor torque for the CWI

$240(\mathrm{P}<0.05)$ and CAT $(\mathrm{P}<0.05)$ groups but not for knee flexor torque $(\mathrm{p}>0.05$; Table 4$)$.

241 However, a time $\mathrm{x}$ group interaction effect was not found for any of these parameters $(\mathrm{p}>0.05)$.

242 Effect size calculations between groups only showed small to trivial differences following the

243 treatment (Table 4). However, CK, muscle soreness and muscle fatigue were moderately larger

244 for the CWI compared to the CAT group at T72.

$245 * * *$ Table 3 around here***

$246 * * *$ Table 4 around here***

\section{Thermal comfort}

249 A main effect of time was found for thermal comfort $(\mathrm{P}<0.01)$ with a time $\mathrm{x}$ group interaction

250 effect $(\mathrm{P}<0.05$; Figure 3$)$. Specifically, time points on the $3^{\text {rd }}$ minute $(2.43 \pm 0.42)$ was

251 significantly greater than the $6^{\text {th }}(1.90 \pm 0.31), 9^{\text {th }}(1.45 \pm 0.41)$ and $12^{\text {th }}(1.35 \pm 0.17)$ minute. No

252 main effect for group was observed $(\mathrm{P}=0.536)$.

***Figure 3 around here***

Discussion

256 Whilst there were no statistical between-group differences, the ES analyses showed greater

257 power output measures and total work done during cycling following the CWI compared to the

258 CAT group with moderate to large differences at immediately post, 24, 48 and 72 hours post

259 strength training session. However, there were small differences between the CWI and CAT 
260 groups for indirect markers of muscle damage (i.e. CK, muscle soreness and muscle force

261 generation capacity) at immediately post, 24,48 and 72 hours post strength training sessions.

262 Whilst CWI only induced trivial effects on indirect markers of muscle damage, the moderate to

263 large differences between the CWI and CAT groups for maximal cycling performance suggest

264 that the combination of cold temperature and water submersion (i.e. CWI), than cold temperature

265 alone (i.e. CAT), may alleviate fatigue and improve the quality of subsequent maximal sprint-

266 based training sessions several days following strength training. In fact, a recent study showed

267 that post-exercise cooling enhanced signalling pathways essential for mitochondrial biogenesis

268 via the AMP-activated protein kinase (AMPK) and p38 mitogen-activated protein kinase

269 (MAPK) in human skeletal muscle (Ihsan et al., 2015). Accordingly, in addition to recovery of

270 neuromuscular properties to improve sprint performance capacity which would remodel skeletal

271 muscle to a more oxidative phenotype (Cochran et al., 2014), CWI appears to activate signalling

272 pathways that may enhance training adaptation. However, chronic post-exercise cooling has also

273 been shown to impair anabolic signalling that orchestrates strength training adaptation which is

274 also paramount for improving sprint-ability (Saez de Villarreal, Requena, Izquierdo, \&

275 Gonzalez-Badillo, 2013). Subsequently, further research is warranted to confirm whether CWI-

276 induced recovery from strength training influences long-term sprint-based anaerobic

277 development.

278 In the current study, power output did not change across time points with the only significant

279 difference between T0 and T72. However, peak power output recovered better at T24, T48 and

$280 \mathrm{~T} 72$ for the CWI (4.0\%, $5.0 \%$ and $6.0 \%$, respectively) than the CAT $(-5.0 \%,-2.0 \%$ and $-2.0 \%$,

281 respectively) group and mean power recovered better at T24, T48 and T72 for the CWI $(2.0 \%$,

$2821.0 \%$ and $2.0 \%$, respectively) than the CAT $(-3.0 \%,-3.0 \%$ and $0.0 \%$, respectively) group with 
283 moderate to large between-group differences. It is well known that a pressure gradient exists

284 within the body, where blood and interstitial fluids flow from high to low pressure environments

285 (Rutkowski \& Swartz, 2007). Besides the beneficial effects of cooling, the purpose of CWI has

286 long been to create a high pressure environment within the area of the body which has undergone

287 muscle damage to increase removal of metabolites to areas of lower pressure (White \& Wells,

288 2013). Accordingly, the greater improvement in anaerobic capacity for the CWI compared to the

289 CAT group in the current study suggests that hydrostatic pressure from water immersion appears

290 to be a strong contributor to accelerating recovery than temperature alone. Indeed, including

291 other experimental groups in different temperature and hydrostatic conditions would have further

292 confirmed this and is considered a limitation in the study. Future research should compare

293 recovery dynamics of CWI and CAT groups with groups in thermoneutral water and air

294 conditions following lower body strength exercises.

295 The improved anaerobic power in the CWI compared to the CAT group in the current study

296 confirm those of Vaile and colleagues (2008) who reported improvement in vertical jump

297 performance in the CWI group compared to a non-hydrotherapy group (i.e. passive recovery) at

29848 and 72 hours post strength exercises. However, there are methodological differences that

299 should be elaborated upon. Vaile and colleagues (2008) determined power output from a single

300 squat jump performance following a single exercise (i.e. leg-press) and incorporated a

301 comparative group in thermoneutral conditions (i.e. warmer than CWI). Conversely, the current

302 study examined maximal cycling performance following a strength training session consisting of

303 multiple exercises (i.e. leg-press, leg extension and leg curl) with a non-hydrotherapy group in

304 temperature conditions equivalent to the CWI group. Given that strength training volume (Doma

305 et al., 2014) and hydrostatic pressure (Leeder et al., 2012) have been shown to affect acute 
306 physiological responses, proper comparisons between the current study and that by Vaile et al

307 (2008) is at present difficult. Nonetheless, the current results extend those of Vaile and

308 colleagues (2008) that CWI may improve lower body power output to a greater degree than non-

309 hydrotherapy following lower body strength exercises.

310 Other than the current study, there has only been one study that has examined the effects of CWI

311 on power output measures several days following a typical lower body strength training session

312 consisting of multiple exercises (Jajtner et al., 2015) as far as we are aware. Interestingly, this

313 study showed that the decrement in lower body power output were similar 24 and 48 hours

314 following strength training between the CWI (-11.4\% and $-12.1 \%$, respectively) and passive

315 recovery $(-11.0 \%$ and $-6.7 \%$, respectively) group which conflict with the current results. The

316 discrepancies in these findings could be attributed to a number of factors. Firstly, Jajtner and

317 colleagues (2015) examined power output during squatting exercises at sub-maximal intensities

318 with greater volume (i.e. three sets $\mathrm{x} \sim 10$ repetitions of squatting exercises at $80 \%$ of $1 \mathrm{RM}$ ).

319 Whilst this method of assessment is important to demonstrate performance during a strength

320 training session, sub-maximal squatting exercises may not be as responsive to fatigue as a single

321 bout of maximal cycling performance assessment as that conducted in the current study.

322 Secondly, Jajtner and colleagues (2015) incorporated strength-trained individuals with an

323 average strength training history of 6.5 years. Conversely, the current study incorporated

324 endurance-trained individuals who had limited exposure to strength training. Subsequently,

325 individuals in the current study may have experienced greater benefits from CWI, particularly on

326 muscular performance, due to their lesser efficient recovery dynamics compared to individuals

327 from the study by Jajtner et al (2015). This conjecture has been suggested by Poppendieck and

328 colleauges (2013) where untrained individuals benefit from CWI to a greater degree due to 
329 potentially larger fatigue and soreness from reduced level of fitness. Furthermore, initial 330 exposure to a typical strength training session has been shown to protect against muscle damage

331 from the second session in strength-untrained men (Doma et al., 2015), known as the repeated

332 bout effect. Accordingly, CWI may be more beneficial during the very initial stages of a

333 concurrent training program for strength-untrained individuals where muscle damage is often

334 excessive due to unaccustomed strength training exercises.

335 The CK measures between groups were comparable despite between-group differences shown

336 for the maximal cycling performance measures. However, this discrepancy in trends between CK 337 and dynamic performance measures following muscle-damaging protocols have actually been 338 reported by others (Chen \& Hsieh, 2001; Chen, Nosaka, Lin, Chen, \& Wu, 2009; Chen, Nosaka, $339 \& \mathrm{Tu}, 2007)$. Furthermore, previous studies have shown that blood levels of intramuscular 340 proteins correlate poorly with changes in muscle function (Clarkson, Byrnes, McCormick, 341 Turcotte, \& White, 1986; Newham, Jones, \& Clarkson, 1987; Newham, Jones, \& Edwards, 342 1983). According to Warren and colleagues (1999) from their extensive narrative review on the 343 appropriate measurement tools for muscle damage markers, they indicated that blood biomarkers 344 do not accurately reflect conditions of the musculature given that the level of intramuscular 345 proteins in the systemic system may be representative of these compounds being released into 346 the blood as well as being removed. Subsequently, Warren and colleagues (1999) suggested 347 MVC as a better indicator of muscle damage than the measurement of intramuscular proteins 348 alone. This further highlights the need to incorporate a range of indirect markers of muscle 349 damage when monitoring the overarching condition of the musculature. Whilst every effort was 350 made to account for potential gender differences, caution should be taken when interpreting our 351 findings given that there was an interaction effect between $\mathrm{CK}$ and gender as a covariate. 
352 Furthermore, previous studies have reported gender differences in exercise-induced muscle

353 damage and pain (Dannecker et al., 2012; Fernandez-Gonzalo, Lundberg, Alvarez-Alvarez, \& de

354 Paz, 2014).

355 Interestingly, when incorporating MVC in conjunction with CK in the current study, similar

356 between-group trends were observed for these two indirect markers of muscle damage despite

357 differences in maximal cycling performance measures. These findings are in line with others,

358 where the trend in MVC was not reflective of changes in running performance measures

359 following muscle-damaging exercises (Chen et al., 2009; Chen et al., 2007; Doma \& Deakin,

360 2014) and also agree with others that have reported no differences between CWI and passive

361 recovery for isometric strength measures (Howatson, Goodall, \& van Someren, 2009; Sellwood,

362 Brukner, Williams, Nicol, \& Hinman, 2007). Given that MVC in the current study was limited to

363 examining contractile properties of the quadriceps and hamstrings, these measures may not have

364 been a true reflection of the mechanics required for maximal cycling performance that requires

365 multiple muscle groups (e.g. quadriceps, hamstrings, gluteal muscles and gastrocnemi).

366 Therefore, greater improvement in maximal cycling performance as a result of CWI may have

367 occurred due to better recovery of muscle contractile properties required for cycling that were

368 not examined in the current study. Further research is warranted to determine whether recovery

369 dynamics other than knee extensor/flexor musculature would contribute to improvement in

370 anaerobic power as a result of CWI.

371 Leeder and colleagues (2012) also reported CWI to induce greater improvement in power output

372 measures but not on strength measures based on a meta-analysis of several CWI studies. The

373 authors speculated that CWI may accelerate recovery of type 2 muscle fibres given that these

374 fibre types are preferentially damaged as a result of strenuous exercises. However, Vaile and 
375 colleagues (2008) reported greater improvement in isometric squat performance and CK 48 and

37672 hours following strength training for the CWI compared to the passive recovery group. The

377 discrepancies in findings between the current study and that by Vaile and colleagues (2008)

378 could be attributed to the CWI protocol. The only discernible difference was the depth of CWI

379 where Vaile et al (2008) had participants immerse in CWI until the clavicle (i.e. entire trunk)

380 compared to the current study where participants were immersed to the iliac crest. By exposing

381 the torso to the CWI there may be an increased removal of metabolites and waste from the lower

382 extremities to the thoracic cavity allowing for an increased rate of muscular regeneration in the

383 affected muscles (Versey et al., 2013).

Conclusion

Overall, the current trends indicated that the application of CWI aided in the recovery of maximal cycling performance in strength-untrained but moderately endurance-trained individuals. These findings suggest that CWI may minimise the detrimental effects of lower body strength training-induced fatigue on the quality of subsequent high intensity intermittent training sessions, particularly during a concurrent training program. Given that strength training could induce sub-optimal training adaptations for modes of endurance exercise (Dolezal \& Potteiger, 1998; Psilander, Frank, Flockhart, \& Sahlin, 2015; Schumann et al., 2015), improving anaerobic capacity via CWI may maximise training adaptations during concurrent training. This speculation could be confirmed by applying CWI between strength training and high intensity intermittent training sessions during a chronic training study (e.g. 10-20 weeks). However, caution should be taken given that speculation for the effects optimising training adaptation 
397

398

399

400

401

402

403

404

405

406

407

408

409

410

411

412

413

414

415

416

417

418

419

420

421

422

423

424

425

426

427

428

429

using CWI has been based solely on one bout of maximal cycling performance. Furthermore, future research should elucidate whether similar findings would be observed in anaerobically

trained individuals and on other modes of exercise performance measures (e.g. running, rowing

or swimming) to expand the practical application of cold-induced recovery methods. Finally,

considering that typical CAT is set at extreme cold conditions with a much lower exposure

window (i.e. under $-100^{\circ} \mathrm{C}$ for $2-3$ minutes) possibly due to conductive/convective properties of

the fluids, it would be interesting to compare intra-muscular temperature between CAT and CWI

conditions similar to that in the current study.

\section{References}

Baechle, T. R., \& Earle, R. W. (2008). Essentials of strength training and conditioning (3rd ed.). Champaign, IL: Human Kinetics.

Byrne, C., \& Eston, R. (2002). Maximal-intensity isometric and dynamic exercise performance after eccentric muscle actions. J Sports Sci, 20(12), 951-959. doi: 10.1080/026404102321011706

Chen, T. C., \& Hsieh, S. S. (2001). Effects of a 7-day eccentric training period on muscle damage and inflammation. Medicine \& Science in Sports \& Exercise, 33(10), 1732-1738.

Chen, T. C., Nosaka, K., Lin, M. J., Chen, H. L., \& Wu, C. J. (2009). Changes in running economy at different intensities following downhill running. J Sports Sci, 27(11), 1137-1144. doi: 914237246 [pii]

10.1080/02640410903062027

Chen, T. C., Nosaka, K., \& Tu, J. H. (2007). Changes in running economy following downhill running. J Sports Sci, 25(1), 55-63. doi: U3K108U025T77N61 [pii]

10.1080/02640410600718228

Clarkson, P. M., Byrnes, W. C., McCormick, K. M., Turcotte, L. P., \& White, J. S. (1986). Muscle soreness and serum creatine kinase activity following isometric, eccentric, and concentric exercise. Int J Sports Med, 7(3), 152-155. doi: 10.1055/s-2008-1025753

Cochran, A. J., Percival, M. E., Tricarico, S., Little, J. P., Cermak, N., Gillen, J. B., . . . Gibala, M. J. (2014). Intermittent and continuous high-intensity exercise training induce similar acute but different chronic muscle adaptations. Exp Physiol, 99(5), 782-791. doi: 10.1113/expphysiol.2013.077453

Cochrane, D. (2004). Alternating hot and cold water immersion for athlete recovery: a review. . Physical Therapy in Sport, 5, 26-32.

Cohen, J. (1988). Statistical power analysis for the behavioral sciences. Hillsdale, New Jersey: Lawrence Erlbaum Associates. 
430

431

432

433

434

435

436

437

438

439

440

441

442

443

444

445

446

447

448

449

450

451

452

453

454

455

456

457

458

459

460

461

462

463

464

465

466

467

468

469

470

471

472

473

474

475

Costello, J. T., Baker, P. R., Minett, G. M., Bieuzen, F., Stewart, I. B., \& Bleakley, C. (2015). Whole-body cryotherapy (extreme cold air exposure) for preventing and treating muscle soreness after exercise in adults. Cochrane Database Syst Rev, 9, CD010789. doi: 10.1002/14651858.CD010789.pub2

Dannecker, E. A., Liu, Y., Rector, R. S., Thomas, T. R., Fillingim, R. B., \& Robinson, M. E. (2012). Sex differences in exercise-induced muscle pain and muscle damage. J Pain, 13(12), 1242-1249. doi: 10.1016/j.jpain.2012.09.014

Dolan, M. G., Thornton, R. M., Fish, D. R., \& Mendel, F. C. (1997). Effects of cold water immersion on edema formation after blunt injury to the hind limbs of rats. J Athl Train, 32(3), 233-237.

Dolezal, B. A., \& Potteiger, J. A. (1998). Concurrent resistance and endurance training influence basal metabolic rate in nondieting individuals. Journal of Applied Physiology, 85(2), 695-700.

Doma, K., \& Deakin, G. (2015). The Acute Effect of Concurrent Training on Running Performance Over 6 Days. Res Q Exerc Sport, 1-10. doi: 10.1080/02701367.2015.1053104

Doma, K., \& Deakin, G. B. (2013a). The effects of combined strength and endurance training on running performance the following day. International Journal of Sport and Health Science, 11, 1-9.

Doma, K., \& Deakin, G. B. (2013b). The effects of strength training and endurance training order on running economy and performance. Appl Physiol Nutr Metab, 38(6), 651-656. doi: 10.1139/apnm-2012-0362

Doma, K., \& Deakin, G. B. (2014). The acute effects intensity and volume of strength training on running performance. Eur J Sport Sci, 14(2), 107-115. doi: 10.1080/17461391.2012.726653

Doma, K., Schumann, M., Sinclair, W. H., Leicht, A. S., Deakin, G. B., \& Hakkinen, K. (2015). The repeated bout effect of typical lower body strength training sessions on sub-maximal running performance and hormonal response. European Journal of Applied Physiology \& Occupational Physiology, 115(8), 1789-1799. doi: 10.1007/s00421-015-3159-z

Fernandez-Gonzalo, R., Lundberg, T. R., Alvarez-Alvarez, L., \& de Paz, J. A. (2014). Muscle damage responses and adaptations to eccentric-overload resistance exercise in men and women. European Journal of Applied Physiology \& Occupational Physiology, 114(5), 1075-1084. doi: 10.1007/s00421-014-2836-7

Hickson, R. C. (1980). The interference effects of training for strength and endurance simulaneously. European Journal of Applied Physiology and Occupational Physiology, 45(2-3), 255-263.

Howatson, G., Goodall, S., \& van Someren, K. A. (2009). The influence of cold water immersions on adaptation following a single bout of damaging exercise. European Journal of Applied Physiology \& Occupational Physiology, 105(4), 615-621. doi: 10.1007/s00421-008-0941-1

Ihsan, M., Markworth, J. F., Watson, G., Choo, H. C., Govus, A., Pham, T., .. . Abbiss, C. R. (2015). Regular postexercise cooling enhances mitochondrial biogenesis through AMPK and p38 MAPK in human skeletal muscle. Am J Physiol Regul Integr Comp Physiol, 309(3), R286-294. doi: 10.1152/ajpregu.00031.2015

Jajtner, A. R., Hoffman, J. R., Gonzalez, A. M., Worts, P. R., Fragala, M. S., \& Stout, J. R. (2015). Comparison of the effects of electrical stimulation and cold-water immersion on muscle soreness after resistance exercise. Journal of Sport Rehabilitation, 24(2), 99-108. doi: 10.1123/jsr.2013-0113

Karunakara, R. G., Lephart, S. M., \& Pincivero, D. M. (1999). Changes in forearm blood flow during single and intermittent cold application. Journal of Orthopaedic \& Sports Physical Therapy, 29(3), 177180. doi: 10.2519/jospt.1999.29.3.177

Kowal, M. A. (1983). Review of physiological effects of cryotherapy. Journal of Orthopaedic \& Sports Physical Therapy, 5(2), 66-73. doi: 10.2519/jospt.1983.5.2.66 
476

477

478

479

480

481

482

483

484

485

486

487

488

489

490

491

492

493

494

495

496

497

498

499

500

501

502

503

504

505

506

507

508

509

510

511

512

513

514

515

516

517

518

519

520

521

522

523

Leeder, J., Gissane, C., van Someren, K., Gregson, W., \& Howatson, G. (2012). Cold water immersion and recovery from strenuous exercise: a meta-analysis. Br J Sports Med, 46(4), 233-240. doi: 10.1136/bjsports-2011-090061

Minett, G. M., Duffield, R., Billaut, F., Cannon, J., Portus, M. R., \& Marino, F. E. (2013). Cold-water immersion decreases cerebral oxygenation but improves recovery after intermittent-sprint exercise in the heat. Scand J Med Sci Sports. doi: 10.1111/sms.12060

Newham, D. J., Jones, D. A., \& Clarkson, P. M. (1987). Repeated high-force eccentric exercise: effects on muscle pain and damage. Journal of Applied Physiology, 63(4), 1381-1386.

Newham, D. J., Jones, D. A., \& Edwards, R. H. (1983). Large delayed plasma creatine kinase changes after stepping exercise. Muscle Nerve, 6(5), 380-385. doi: 10.1002/mus.880060507

Nieman, D. C., Gillitt, N. D., Shanely, R. A., Dew, D., Meaney, M. P., \& Luo, B. (2014). Vitamin D2 supplementation amplifies eccentric exercise-induced muscle damage in NASCAR pit crew athletes. Nutrients, 6(1), 63-75. doi: 10.3390/nu6010063

Poppendieck, W., Faude, O., Wegmann, M., \& Meyer, T. (2013). Cooling and performance recovery of trained athletes: a meta-analytical review. Int J Sports Physiol Perform, 8(3), 227-242.

Psilander, N., Frank, P., Flockhart, M., \& Sahlin, K. (2015). Adding strength to endurance training does not enhance aerobic capacity in cyclists. Scand J Med Sci Sports, 25(4), e353-359. doi: $10.1111 /$ sms. 12338

Rhea, M. R. (2004). Determining the magnitude of treatment effects in strength training research through the use of the effect size. Journal of Strength \& Conditioning Research, 18(4), 918-920. doi: $10.1519 / 14403.1$

Roberts, L. A., Nosaka, K., Coombes, J. S., \& Peake, J. M. (2014). Cold water immersion enhances recovery of submaximal muscle function after resistance exercise. Am J Physiol Regul Integr Comp Physiol, 307(8), R998-R1008. doi: 10.1152/ajpregu.00180.2014

Rowsell, G. J., Reaburn, P., Toone, R., Smith, M., \& Coutts, A. J. (2014). Effect of run training and coldwater immersion on subsequent cycle training quality in high-performance triathletes. Journal of Strength \& Conditioning Research, 28(6), 1664-1672. doi: 10.1519/JSC.0000000000000455

Rutkowski, J. M., \& Swartz, M. A. (2007). A driving force for change: interstitial flow as a morphoregulator. Trends Cell Biol, 17(1), 44-50. doi: 10.1016/j.tcb.2006.11.007

Saez de Villarreal, E., Requena, B., Izquierdo, M., \& Gonzalez-Badillo, J. J. (2013). Enhancing sprint and strength performance: combined versus maximal power, traditional heavy-resistance and plyometric training. J Sci Med Sport, 16(2), 146-150. doi: 10.1016/j.jsams.2012.05.007

Schaal, K., Y, L. E. M., Louis, J., Filliard, J. R., Hellard, P., Casazza, G., \& Hausswirth, C. (2015). Whole-Body Cryostimulation Limits Overreaching in Elite Synchronized Swimmers. Medicine \& Science in Sports \& Exercise, 47(7), 1416-1425. doi: 10.1249/MSS.0000000000000546

Schumann, M., Mykkanen, O. P., Doma, K., Mazzolari, R., Nyman, K., \& Hakkinen, K. (2015). Effects of endurance training only versus same-session combined endurance and strength training on physical performance and serum hormone concentrations in recreational endurance runners. Appl Physiol Nutr Metab, 40(1), 28-36. doi: 10.1139/apnm-2014-0262

Sellwood, K. L., Brukner, P., Williams, D., Nicol, A., \& Hinman, R. (2007). Ice-water immersion and delayed-onset muscle soreness: a randomised controlled trial. Br J Sports Med, 41(6), 392-397. doi: 10.1136/bjsm.2006.033985

Tabata, I., Nishimura, K., Kouzaki, M., Hirai, Y., Ogita, F., Miyachi, M., \& Yamamoto, K. (1996). Effects of moderate-intensity endurance and high-intensity intermittent training on anaerobic capacity and VO2max. Medicine \& Science in Sports \& Exercise, 28(10), 1327-1330.

Twist, C., \& Eston, R. (2005). The effects of exercise-induced muscle damage on maximal intensity intermittent exercise performance. European Journal of Applied Physiology \& Occupational Physiology, 94(5-6), 652-658. doi: 10.1007/s00421-005-1357-9 
524

525

526

527

528

529

530

531

532

533

534

535

536

537

538

539

540

541

542
Vaile, J., Halson, S., Gill, N., \& Dawson, B. (2008). Effect of hydrotherapy on the signs and symptoms of delayed onset muscle soreness. European Journal of Applied Physiology \& Occupational Physiology, 102(4), 447-455. doi: 10.1007/s00421-007-0605-6

Versey, N. G., Halson, S. L., \& Dawson, B. T. (2013). Water immersion recovery for athletes: effect on exercise performance and practical recommendations. Sports Med, 43(11), 1101-1130. doi: 10.1007/s40279-013-0063-8

Warren, G. L., Lowe, D. A., \& Armstrong, R. B. (1999). Measurement tools used in the study of eccentric contraction-induced injury. Sports Med, 27(1), 43-59.

Wells, C., Edwards, A., Fysh, M., \& Drust, B. (2014). Effects of high-intensity running training on soccerspecific fitness in professional male players. Appl Physiol Nutr Metab, 39(7), 763-769. doi: 10.1139/apnm-2013-0199

White, G. E., \& Wells, G. D. (2013). Cold-water immersion and other forms of cryotherapy: physiological changes potentially affecting recovery from high-intensity exercise. Extrem Physiol Med, 2(1), 26. doi: 10.1186/2046-7648-2-26

Ziemann, E., Olek, R. A., Grzywacz, T., Kaczor, J. J., Antosiewicz, J., Skrobot, W., . . Laskowski, R. (2014). Whole-body cryostimulation as an effective way of reducing exercise-induced inflammation and blood cholesterol in young men. Eur Cytokine Netw, 25(1), 14-23. doi: 10.1684/ecn.2014.0349 


\section{Table $\mathbf{1}$ (on next page)}

Mean \pm standard deviation for the main effect of time for the maximal cycling performance parameters measured prior to (T0), immediately post (T1), 24 (T24), 48 (T48) and 72 (T72) hours post strength training session

Mean \pm standard deviation for the main effect of time for the maximal cycling performance parameters measured prior to (T0), immediately post (T1), 24 (T24), 48 (T48) and 72 (T72) hours post strength training session 
1 Table 1. Mean \pm standard deviation for the main effect of time for the maximal cycling

2 performance parameters measured prior to (T0), immediately post (T1), 24 (T24), 48 (T48) and

372 (T72) hours post strength training session

\begin{tabular}{llll}
\hline & Mean Power $(\mathrm{W})$ & Peak Power $(\mathrm{W})$ & Total Work $(\mathrm{W})$ \\
\hline T0 & $552.1 \pm 94.25$ & $741.15 \pm 138.06$ & $5079.27 \pm 867.20$ \\
T1 & $543.35 \pm 98.14$ & $693.75 \pm 174.71$ & $4999.34 \pm 903.14$ \\
T24 & $556.45 \pm 96.61^{*}$ & $750.65 \pm 149.05$ & $5119.46 \pm 889.15^{*}$ \\
T48 & $554.8 \pm 98.55^{*}$ & $754.70 \pm 153.13$ & $5104.32 \pm 877.80$ \\
T72 & $562.45 \pm 95.68$ & $765.25 \pm 138.81$ & $5171.77 \pm 880.98^{*}$ \\
\hline
\end{tabular}

$4 *$ Significantly greater than $\mathrm{T} 1(\mathrm{P} \leq 0.05)$

5 


\section{Table 2 (on next page)}

Mean \pm standard deviation for the main effect of time for the maximal cycling performance parameters for the cold water immersion (CWI) and cold air therapy (CAT) group measured prior to (T0), immediately post (T1), 24 (T24), 48 (T48) and 72 (T72) hours $p$

Mean \pm standard deviation for the main effect of time for the maximal cycling performance parameters for the cold water immersion (CWI) and cold air therapy (CAT) group measured prior to (T0), immediately post (T1), 24 (T24), 48 (T48) and 72 (T72) hours post strength training session 
1 Table 2. Mean \pm standard deviation for the main effect of time for the maximal cycling 2 performance parameters for the cold water immersion (CWI) and cold air therapy (CAT) group 3 measured prior to (T0), immediately post (T1), 24 (T24), 48 (T48) and 72 (T72) hours post

4 strength training session

\begin{tabular}{lllllll}
\hline & \multicolumn{2}{l}{ Mean Power $(W)$} & \multicolumn{2}{l}{ Peak Power $(W)$} & \multicolumn{2}{l}{ Total Work (W) } \\
\cline { 2 - 6 } & CWI & CAT & CWI & CAT & CWI & CAT \\
\hline T0 & $541.9 \pm$ & $562.3 \pm$ & $727.7 \pm$ & $754.6 \pm$ & $4985.6 \pm$ & $5172.9 \pm$ \\
& 107.6 & 143.0 & 154.2 & 212.0 & 989.7 & 1316.0 \\
T1 & $534.1 \pm$ & $552.6 \pm$ & $648.1 \pm$ & $739.4 \pm$ & $4913.4 \pm$ & $5085.3 \pm$ \\
& 117.2 & 144.9 & 247.8 & 220.5 & 1078.3 & 1333.4 \\
T24 & $551.3 \pm$ & $561.6 \pm$ & $758.9 \pm$ & $742.4 \pm$ & $5768.8 \pm$ & $5165.9 \pm$ \\
& 113.4 & 144.2 & 185.1 & 214.1 & 1043.7 & 1327.0 \\
T48 & $547.6 \pm$ & $562.0 \pm$ & $762.3 \pm$ & $747.1 \pm$ & $5037.5 \pm$ & $5171.1 \pm$ \\
& 113.1 & 141.5 & 170.2 & 235.8 & 1041.4 & 1301.3 \\
T72 & $553.1 \pm$ & $571.8 \pm$ & $763.2 \pm$ & $767.3 \pm$ & $5087.6 \pm$ & $5255.9 \pm$ \\
& 113.7 & 141.7 & 150.2 & 216.6 & 1044.6 & 1306.4 \\
\hline
\end{tabular}

5 


\section{Table 3 (on next page)}

Mean \pm standard deviation for the main effect of time for creatine kinase (CK), muscle soreness, muscle fatigue, knee extensor torque (KET) and knee flexor torque (KFT) measured prior to (T0), immediately post (T1), 24 (T24), 48 (T48) and 72 (T72) hours $p$

Mean \pm standard deviation for the main effect of time for creatine kinase (CK), muscle soreness, muscle fatigue, knee extensor torque (KET) and knee flexor torque (KFT) measured prior to (T0), immediately post (T1), 24 (T24), 48 (T48) and 72 (T72) hours post strength training session 
1 Table 3. Mean \pm standard deviation for the main effect of time for creatine kinase $(\mathrm{CK})$, muscle 2 soreness, muscle fatigue, knee extensor torque (KET) and knee flexor torque (KFT) measured 3 prior to (T0), immediately post (T1), 24 (T24), 48 (T48) and 72 (T72) hours post strength

4 training session

\begin{tabular}{llllll}
\hline & CK $\left(\mathrm{U}^{-1}\right)$ & Soreness & Fatigue & KET $\left(\mathrm{N} . \mathrm{m}^{-1}\right)$ & KFT $\left(\mathrm{N} . \mathrm{m}^{-1}\right)$ \\
\hline T0 & $116.4 \pm 45.42$ & $4.25 \pm 3.89$ & $4.50 \pm 4.55$ & $199.32 \pm 32.46$ & $52.71 \pm 17.68$ \\
T1 & $252.83 \pm 101.74$ & $39.55 \pm 15.14^{*}$ & $47.55 \pm 16.62^{*}$ & $160.65 \pm 26.83 \dagger$ & $50.51 \pm 15.26$ \\
T24 & $252.83 \pm 101.42 *$ & $47.55 \pm 14.57 *$ & $39.60 \pm 16.88^{*}$ & $178.05 \pm 34.67$ & $52.56 \pm 15.63$ \\
T48 & $173.25 \pm 77.03 *$ & $35.40 \pm 14.60^{*}$ & $27.9 \pm 16.43^{*}$ & $180.92 \pm 36.59$ & $52.85 \pm 16.02$ \\
T72 & $141.65 \pm 50.04$ & $17.50 \pm 8.15^{*}$ & $12.90 \pm 9.29^{*}$ & $183.10 \pm 38.20$ & $53.77 \pm 17.51$ \\
\hline
\end{tabular}

$5 *$ Significantly greater than T0, T48 and T72

$6 * *$ Significantly greater than $\mathrm{T} 0$

$7 \uparrow$ Significantly lower than T0, T24, T48 and T72 


\section{Table 4 (on next page)}

Mean \pm standard deviation of creatine kinase (CK), muscle soreness, muscle fatigue, knee extensor torque (KET) and knee flexor torque (KFT) from pre (T0) to immediately post (T1), 24 hours (T24), 48 hours (T48) and 72 hours (T72) post in the cold water im

Mean \pm standard deviation of creatine kinase $(C K)$, muscle soreness, muscle fatigue, knee extensor torque (KET) and knee flexor torque (KFT) from pre (T0) to immediately post (T1), 24 hours (T24), 48 hours (T48) and 72 hours (T72) post in the cold water immersion (CWI) and cold air therapy (CAT) groups with effect size calculations between groups for each time point 
1 Table 4. Mean \pm standard deviation of creatine kinase $(\mathrm{CK})$, muscle soreness, muscle fatigue, 2 knee extensor torque (KET) and knee flexor torque (KFT) from pre (T0) to immediately post 3 (T1), 24 hours (T24), 48 hours (T48) and 72 hours (T72) post in the cold water immersion (CWI) 4 and cold air therapy (CAT) groups with effect size calculations between groups for each time 5 point

\begin{tabular}{|c|c|c|c|}
\hline Variable & CWI & CAT & Effect size \\
\hline \multicolumn{4}{|c|}{$\mathrm{CK}\left(\mathrm{U} \cdot \mathrm{L}^{-1}\right)$} \\
\hline T0 & $123.62 \pm 75.05$ & $109.18 \pm 42.49$ & 0.25 (small) \\
\hline $\mathrm{T} 24$ & $259.39 \pm 155.39^{*}$ & $246.27 \pm 113.47^{*}$ & 0.10 (trivial) \\
\hline $\mathrm{T} 48$ & $160.46 \pm 106.85^{*}$ & $186.03 \pm 99.88^{*}$ & 0.25 (small) \\
\hline $\mathrm{T} 72$ & $157.88 \pm 81.57$ & $125.41 \pm 48.7$ & 0.50 (moderate) \\
\hline \multicolumn{4}{|c|}{ Muscle soreness } \\
\hline T0 & $3.8 \pm 5.9$ & $4.7 \pm 6.2$ & 0.15 (trivial) \\
\hline $\mathrm{T} 1$ & $39.5 \pm 20.3^{*}$ & $39.6 \pm 24.8^{*}$ & 0.00 (trivial) \\
\hline $\mathrm{T} 24$ & $47 \pm 20.2^{*}$ & $48.1 \pm 23.2 *$ & 0.05 (trivial) \\
\hline $\mathrm{T} 48$ & $40 \pm 20 *$ & $30.8 \pm 19.7^{*}$ & 0.46 (small) \\
\hline $\mathrm{T} 72$ & $20.9 \pm 11.8^{*}$ & $14.1 \pm 9.8^{*}$ & 0.63 (moderate) \\
\hline \multicolumn{4}{|c|}{ Muscle fatigue } \\
\hline T0 & $4.7 \pm 7.4$ & $4.3 \pm 6.7$ & 0.06 (trivial) \\
\hline $\mathrm{T} 1$ & $54 \pm 18.5^{*}$ & $41.1 \pm 26^{*}$ & 0.58 (moderate) \\
\hline $\mathrm{T} 24$ & $44 \pm 21.3^{*}$ & $35.2 \pm 23.9^{*}$ & 0.39 (small) \\
\hline $\mathrm{T} 48$ & $32 \pm 22.3^{*}$ & $23.8 \pm 21.1 *$ & 0.38 (small) \\
\hline T72 & $16.4 \pm 14.2^{*}$ & $9.4 \pm 9.6$ & 0.59 (moderate) \\
\hline \multicolumn{4}{|c|}{$\operatorname{KET}\left(\mathrm{N}^{\prime} \mathrm{m}^{-1}\right)$} \\
\hline T0 & $203.7 \pm 52.1$ & $192.7 \pm 49.6$ & 0.15 (trivial) \\
\hline $\mathrm{T} 1$ & $162.3 \pm 39.6^{* *}$ & $156.8 \pm 41.7 * *$ & 0.00 (trivial) \\
\hline $\mathrm{T} 24$ & $180.8 \pm 52.8$ & $174.9 \pm 53.7 * *$ & 0.05 (trivial) \\
\hline $\mathrm{T} 48$ & $190.0 \pm 58.6$ & $180.4 \pm 57.3 * *$ & 0.46 (small) \\
\hline $\mathrm{T} 72$ & $190.8 \pm 59.5$ & $183.1 \pm 57.6^{* *}$ & 0.63 (moderate) \\
\hline \multicolumn{4}{|c|}{$\operatorname{KFT}\left(\mathrm{N}^{\prime} \mathrm{m}^{-1}\right)$} \\
\hline T0 & $51.5 \pm 19.6$ & $48.2 \pm 19.1$ & 0.06 (trivial) \\
\hline $\mathrm{T} 1$ & $48.9 \pm 20.3$ & $45.7 \pm 19.3$ & 0.58 (moderate) \\
\hline $\mathrm{T} 24$ & $50.1 \pm 19.7$ & $46.7 \pm 19.4$ & 0.39 (small) \\
\hline T48 & $51.9 \pm 20.7$ & $47.9 \pm 20.1$ & 0.38 (small) \\
\hline $\mathrm{T} 72$ & $53.4 \pm 24.8$ & $50.8 \pm 25.0$ & 0.59 (moderate) \\
\hline
\end{tabular}

* Significantly greater than T0 $(\mathrm{P}<0.05)$

* Significantly less than T0 $(\mathrm{P}<0.05)$ 
Figure 1 (on next page)

Schematic of research design

Schematic of the research design including cold water immersion (CWI) group and cold air therapy (CAT) group with indirect markers of muscle damage (i.e. creatine kinase, isometric knee flexor/extensor torque, muscle soreness and muscle fatigue) and anaerobic performance (i.e. cycling Wingate test) denoted in vertical arrows conducted prior to (T0), immediately post (T1), 24 (T24), 48 (T48) and 72 (T72) hours post strength training (ST) session 


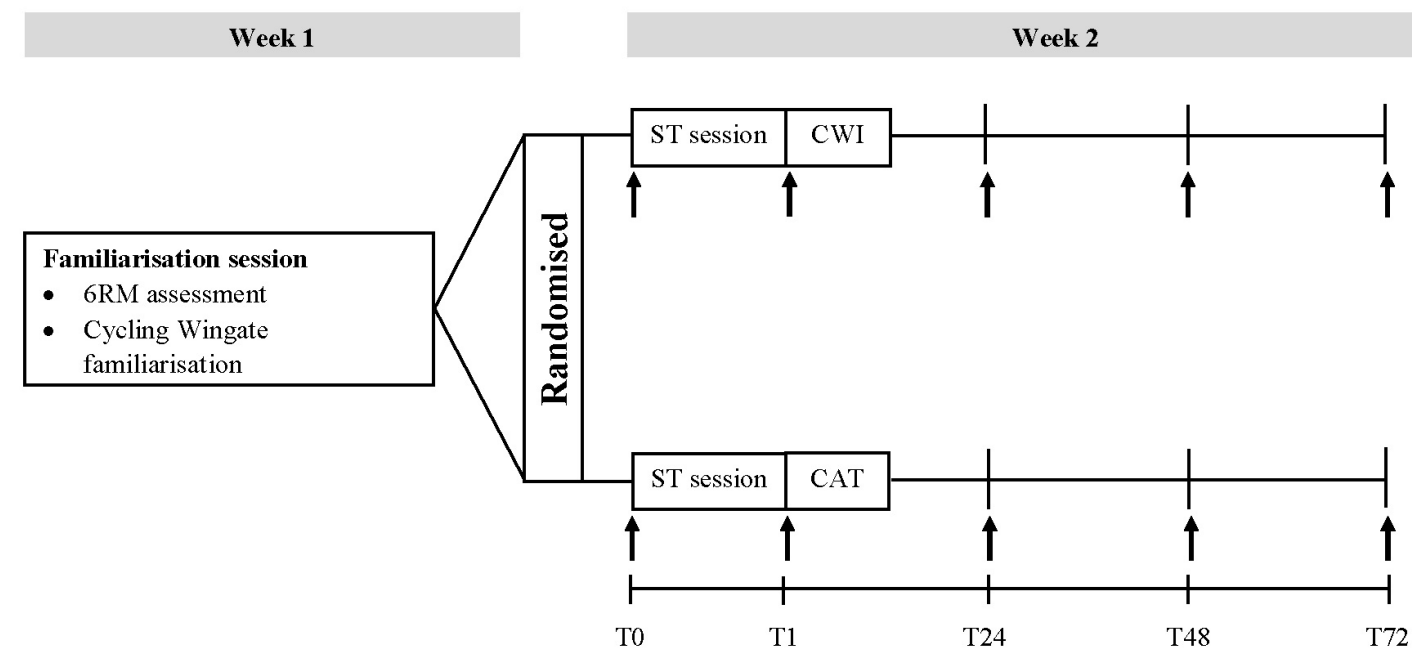


Figure 2 (on next page)

Percentage change in cycling performance

Percentage change in (a) peak watts, (b) mean watts and (c) total work for the cold water immersion (CWI) and cold air therapy (CAT) groups with effect size calculations between groups shown above bar graphs at immediate post (T1), 24 hours (T24), 48 hour *Indicates significant increase from post $(p \leq 0.05)$ 

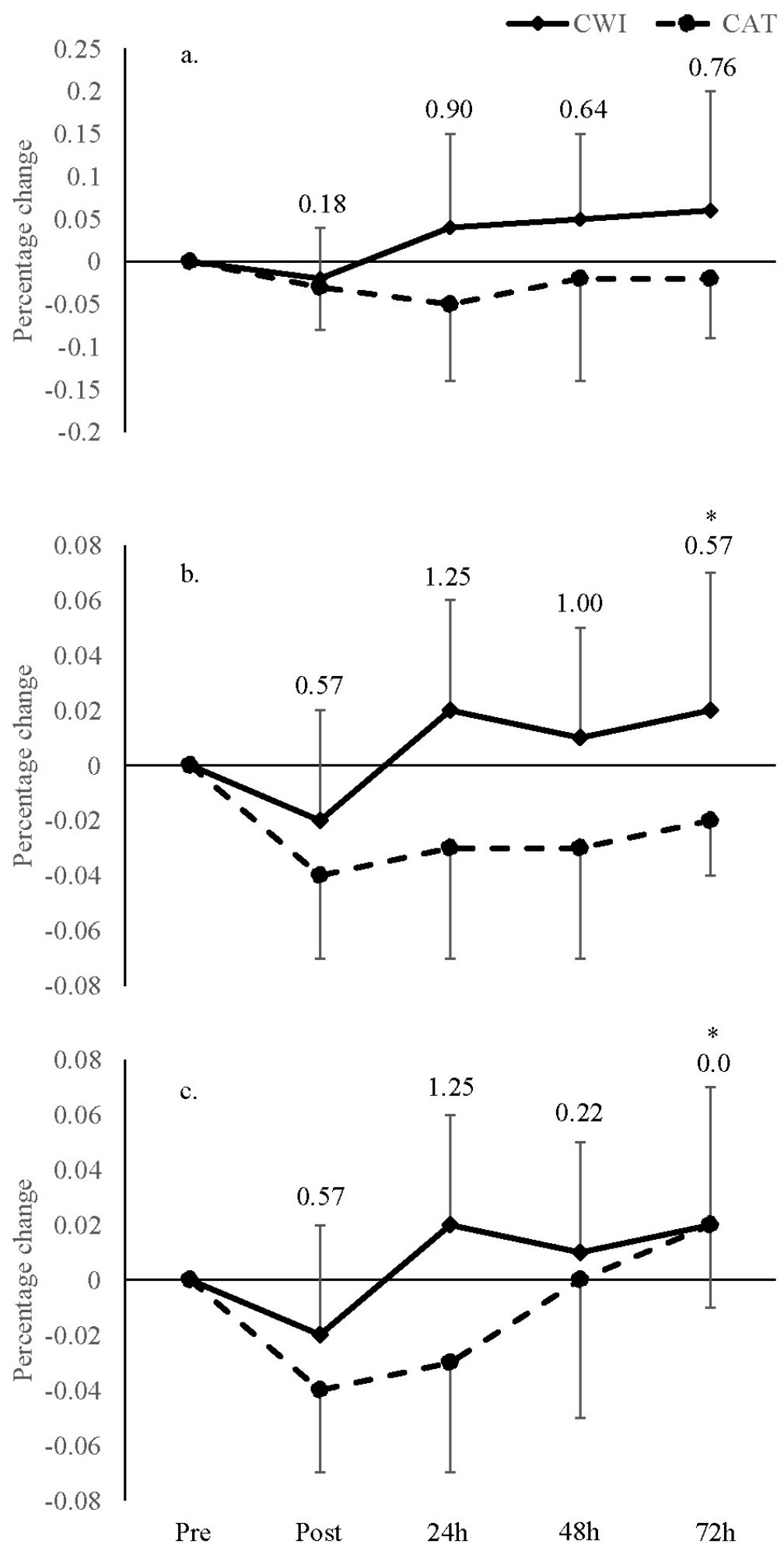


\section{Figure 3 (on next page)}

Thermal comfort

Thermal comfort for the cold water immersion (CWI) and cold air therapy (CAT) groups during the recovery interventions $*$ Indicates significant decrease from the $3^{\text {rd }}$ minute 


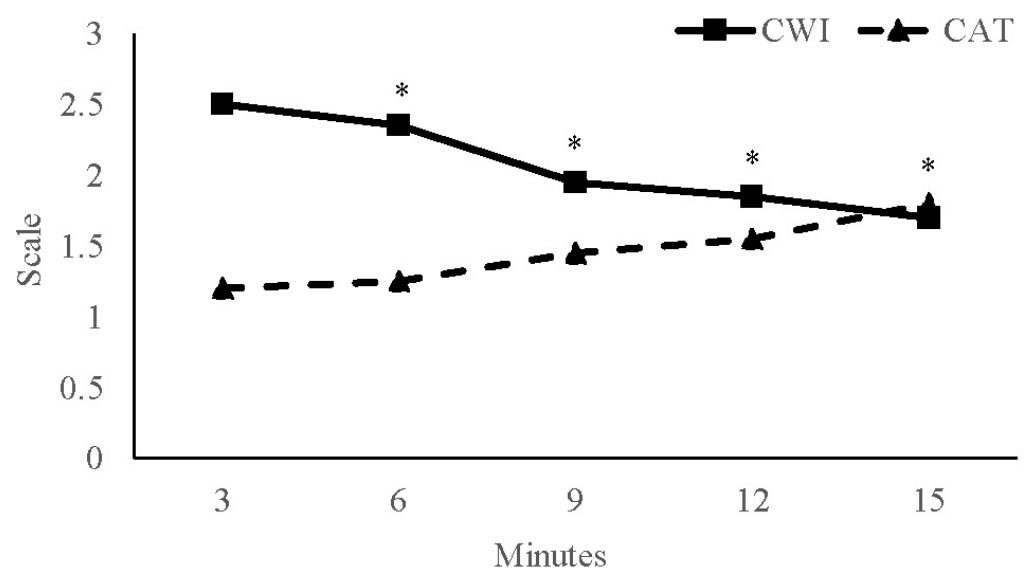

